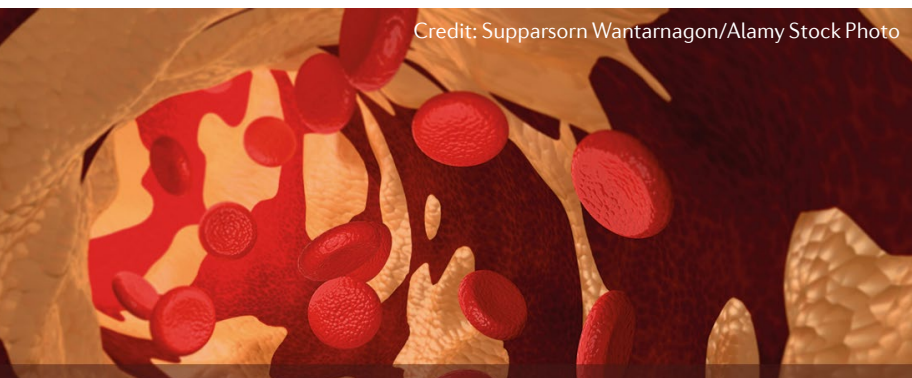

$\Rightarrow$ SYSTEMTC LUPUS ERYTHEMATOSUS

\section{IL-27 links lipids to autoimmunity}

Research published in Nature

Immunology links atherosclerosis

to the pathogenesis of systemic

lupus erythematosus (SLE).

"Atherogenic dyslipidemia increases

the production of autoantibodies,

particularly pathogenic IgG2c, and

severity of SLE by enhancing

T follicular helper cell responses,"

says corresponding author of the new

study, Yeonseok Chung.

IL-27 increases antibody

production by facilitating germinal

centre reactions in secondary

lymphoid organs, and excessive

activation of this pathway is associated with autoantibody production and pathogenesis of SLE. The new study shows (in mice) that a major source of IL-27 production is dendritic cells (DCs) that are responding to serum lipids via Toll-like receptor 4 (TLR4) signalling.

Chung's team transferred bone marrow cells from lupus-prone BXD2 mice into either Apoe ${ }^{-/-}$mice or $\mathrm{Ldlr}^{-1-}$ mice (both of which are used to model atherosclerosis), or into wild-type mice as a control $\left(\mathrm{WT}^{\mathrm{BXD} 2}\right)$, and put them on a high-fat diet, to model the effect of atherosclerosis and hyperlipidemia on the severity and progression of SLE. The atherogenic mice had higher anti-dsDNA autoantibody levels than control mice, including a large increase in circulating IgG2c, and more severe glomerulonephritis.

The researchers detected an increase in the number of $\mathrm{T}$ follicular helper $\left(\mathrm{T}_{\mathrm{FH}}\right)$ cells in the atherogenic mice, and flow cytometry and RNA-seq showed that the increase in $\mathrm{T}_{\mathrm{FH}}$ cell numbers was accompanied by an increase in expression of genes associated with helping
B cells produce IgG2c antibodies. Chung's group also noticed a large increase in serum levels of IL-27 in the lupus-prone atherogenic mice (compared with $\mathrm{WT}^{\mathrm{BDX} 2}$ mice), and that CD $11 b^{+}$DCs from these mice produce more IL-27.

Using $\mathrm{Il}_{27^{-/-}}$mice and $\mathrm{Tlr} 4^{-1-}$ mice they established that TLR4stimulatory lipids such as oxidized LDL and long chain saturated fatty acids can increase IL-27 production by the CD11b+ DC subset.

To demonstrate the clinical relevance of these findings, the researchers present data showing that patients with high serum LDL and total cholesterol levels (but not diagnosed with SLE) also have high levels of serum IL-27 and anti-dsDNA autoantibodies. "This hyperlipidemiaTLR4-IL27-CXCR3 ${ }^{+} \mathrm{T}_{\mathrm{FH}}$ cell axis might explain the tight association between atherosclerosis and SLE in humans," concludes Chung.

Nicholas J. Bernard

ORIGINAL ARTICLE Ryu, H. et al. Atherogenic dyslipidemia promotes autoimmune follicular helper T cell responses via IL-27. Nat. Immunol. https://doi.org/10.1038/s41590-018-0102-6 (2018)
Oestrogen

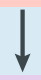

Hormone status regulates autoantibody pathogenicity

Oestrogen levels can affect the inflammatory properties of autoantibodies by regulating their glycan composition, according to a new study published in Arthritis Research \& Therapy. The findings could explain why menopause, which is associated with a decrease in oestrogen, coincides with an increase in susceptibility to rheumatoid arthritis (RA).

Previous work has demonstrated that the composition of glycans present in the $\mathrm{Fc}$ region of antibodies (known as IgG-Fc glycans) can regulate antibody effector functions. In particular, a lack of end-terminal sialic acid residues on IgG-Fc glycans increases the pro-inflammatory activity of antibodies, whereas the presence of this residue has anti-inflammatory effects.

To study the link between menopause and antibody responses, Engdahl and

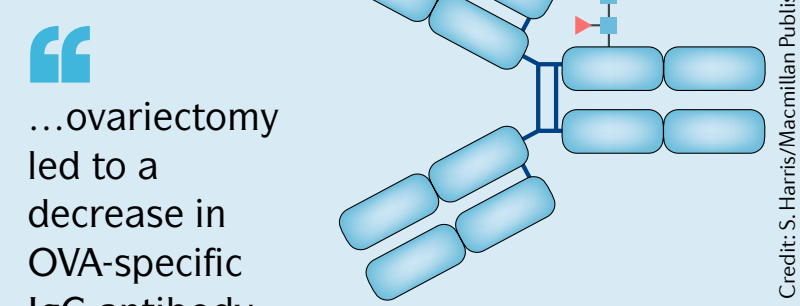

IgG antibody sialylation, which was reversed with oestrogen treatment

colleagues used ovariectomized mice (or sham-operated mice as a control) that were immunized with ovalbumin (OVA). Prior to immunization, the mice were treated with either placebo or oestrogen, modelling the effects of oestrogen deficiency or enzyme hormone-replacement therapy (HRT), respectively.
As expected, OVA immunization led to an increase in total and OVA-specific $\operatorname{lgG}$ antibody levels. The development and affinity of OVA-specific lgG antibodies was unaffected by oestrogen treatment. However, ovariectomy led to a decrease in OVA-specific IgG antibody sialylation, which was reversed with oestrogen treatment.

Oestrogen induced the expression of the sialylation enzyme $\beta$-galactoside a2,6-sialyltransferase 1 (ST6Gal1) in mouse and human plasmablasts. Furthermore, serum antibodies from post-menopausal women with $R A$ receiving HRT had increased levels of sialylation compared with those women with RA not receiving HRT.

These results indicate that oestrogen is a protective factor in RA development, and oestrogen treatment could be beneficial in some patients.

\section{Jessica McHugh}

ORIGINAL ARTICLE Engdahl, C. et al. Estrogen induces St6gal1 expression and increases $\lg G$ sialylation in mice and patients with rheumatoid arthritis: a potential explanation for the increased risk of rheumatoid arthritis in postmenopausal women. Arthritis Res. Ther. https://doi.org/10.1186/ s13075-018-1586-z (2018) 